PROCEEDINGS OF THE

AMERICAN MATHEMATICAL SOCIETY

Volume 134, Number 10, October 2006, Pages 2983-2987

S $0002-9939(06) 08363-8$

Article electronically published on May 4, 2006

\title{
DUALIZED AND SCALED FITZPATRICK FUNCTIONS
}

\author{
STEPHEN SIMONS
}

(Communicated by Jonathan M. Borwein)

\begin{abstract}
In this paper, we obtain an explicit formula for the interior of the domain of a maximal monotone multifunction in terms of its Fitzpatrick function.
\end{abstract}

\section{Preliminaries}

In recent years, there has been considerable interest in the Fitzpatrick function of a maximal monotone multifunction. In this paper, we use a mixed norm $\times$ weak* topology on the product of a Banach space and its dual to obtain an explicit formula for the interior of the domain of a maximal monotone multifunction in terms of its Fitzpatrick function.

A good starting point for our discussion is the result proved by Rockafellar in [5, Theorem 1, p. 398] that the interior of the domain of a maximal monotone multifunction on a real Banach space is convex. This was sharpened in 6, Theorem 18.3 , p. 67], where an explicit description of this interior was given in terms of a convex function defined by an abstract "free convexification" of the graph of the multifunction. If this result is combined with the result of [7, Remark 5.6, pp. 13-14], we obtain an explicit description of this interior in terms of the Fitzpatrick function of the multifunction. So it is natural to ask whether this explicit description can be obtained directly from the Fitzpatrick function without the abstract free convexification. We show how this can be done in Theorem 2.2, where we prove that if $S$ is maximal monotone with Fitzpatrick function $\varphi_{S}$, then int $\left(\operatorname{pr}_{1} \operatorname{dom} \varphi_{S}\right)=$ int $D(S)$. ( $\mathrm{pr}_{1}$ is defined below.) The main stepping stones towards Theorem 2.2 are some results about various operations that one can perform on appropriate convex functions that are lower semicontinuous with respect to a norm $\times$ weak* topology. These results are contained in Section 1, the main result being Theorem 1.3. In a recent paper, Borwein [1, Corollary 9] gives a different approach to Rockafellar's result using the Fitpatrick function.

If $F$ is a real vector space and $f: F \mapsto]-\infty, \infty]$, then we use the standard notation $\operatorname{dom} f:=\{x \in F: f(x) \in \mathbb{R}\} . f$ is said to be proper if $\operatorname{dom} f \neq \emptyset$.

We now assume that $E$ is a nonzero real (not necessarily reflexive) normed space with norm-topology $\mathcal{T}_{\|\|}$, and $E^{*}$ is its topological dual space. We use the notation $\mathrm{pr}_{1}$ to stand for the projection of $E \times E^{*}$ onto $E$. Let $\mathcal{N} \mathcal{W}$ be the $\mathcal{T}_{\|} \times w\left(E^{*}, E\right)$

Received by the editors May 3, 2005.

2000 Mathematics Subject Classification. Primary 47H05; Secondary 26B25.

Key words and phrases. Monotone multifunction, Fitzpatrick function, convex function, conjugate function, Fenchel duality, weak* topology.

(C)2006 American Mathematical Society Reverts to public domain 28 years from publication 2983 
topology on $E \times E^{*}$. Then $\left(E \times E^{*}, \mathcal{N} \mathcal{W}\right)$ is a Hausdorff locally convex space with topological dual $E \times E^{*}$ under the pairing

$$
\left\lfloor\left(x, x^{*}\right),\left(y, y^{*}\right)\right\rfloor:=\left\langle x, y^{*}\right\rangle+\left\langle y, x^{*}\right\rangle .
$$

1. The $\mathcal{T}_{\|} \| \times w\left(E^{*}, E\right)$ TOPOLOGY ON $E \times E^{*}$

We now introduce three functions derived from an appropriate convex function on $E \times E^{*}$ : two defined on $E \times E^{*}$, and the other on $E$.

Definition 1.1. Let $E$ be a nonzero real Banach space and let $f: E \times E^{*} \mapsto$ ]$-\infty, \infty]$ be proper, convex and $\mathcal{N} \mathcal{W}$-lower semicontinuous.

We define the function $\left.\left.f^{\vee}\left(x, x^{*}\right): E \times E^{*} \mapsto\right]-\infty, \infty\right]$ by $f^{\vee}\left(x, x^{*}\right):=f\left(x, x^{*}\right) \vee$ $\left\|x^{*}\right\|$, where $\alpha \vee \beta:=\max \{\alpha, \beta\}$. Then $f^{\vee}\left(x, x^{*}\right)$ is convex and nonnegative on $E \times E^{*}$, and $\operatorname{dom} f^{\vee}=\operatorname{dom} f$.

We define the function $\left.\left.f^{@}: E \times E^{*} \mapsto\right]-\infty, \infty\right]$ by

$$
f^{@}\left(y, y^{*}\right):=\sup _{\left(x, x^{*}\right) \in E \times E^{*}}\left[\left\langle x, y^{*}\right\rangle+\left\langle y, x^{*}\right\rangle-f\left(x, x^{*}\right)\right] .
$$

$f^{@}$ is the $\mathcal{N} \mathcal{W}$-conjugate of $f$. We use this notation to distinguish $f^{@}$ from the norm-conjugate of $f$, which is defined on $E^{*} \times E^{* *}$. Then $f^{@}$ is proper, convex and $\mathcal{N} \mathcal{W}$-lower semicontinuous, and, from the Fenchel-Moreau theorem for locally convex spaces (see Zălinescu, [8, Theorem 2.3.3, pp. 77-78]),

$$
f^{@ @}=f .
$$

We define the function $\left.\left.f^{\div}: E \rightarrow\right]-\infty, \infty\right]$ by

$$
f \div(x):=\sup _{\left(y, y^{*}\right) \in E \times E^{*}} \frac{\left\langle x, y^{*}\right\rangle-f^{@}\left(y, y^{*}\right)}{1+\|y\|} .
$$

Since $f^{\div}$is the supremum of a family of continuous affine functions and $f^{@}$ is proper, $f^{\div}$is proper, convex and lower semicontinuous.

Lemma 1.2. Let $E$ be a nonzero real Banach space, let $\left.\left.f: E \times E^{*} \mapsto\right]-\infty, \infty\right]$ be proper, convex and $\mathcal{N} \mathcal{W}$-lower semicontinuous, and $x \in E$. Then

$$
f^{\doteqdot}(x) \vee 0=\min _{t^{*} \in E^{*}} f^{\vee}\left(x, t^{*}\right) \text {. }
$$

Proof. If $t^{*} \in E^{*}$, then

$$
\left(y, y^{*}\right) \in E \times E^{*} \quad \Longrightarrow \quad\left\langle x, y^{*}\right\rangle+\left\langle y, t^{*}\right\rangle \leq f\left(x, t^{*}\right)+f^{@}\left(y, y^{*}\right) .
$$

Thus

$$
\begin{aligned}
\left(y, y^{*}\right) \in E \times E^{*} \Longrightarrow\left\langle x, y^{*}\right\rangle-f^{@}\left(y, y^{*}\right) & \leq f\left(x, t^{*}\right)+\|y\|\left\|t^{*}\right\| \\
& \leq(1+\|y\|) f^{\vee}\left(x, t^{*}\right) .
\end{aligned}
$$

It follows by dividing by $1+\|y\|$ and taking the supremum over $\left(y, y^{*}\right) \in E \times E^{*}$ that $f^{\div}(x) \leq f^{\vee}\left(x, t^{*}\right)$. It is immediate from this (and the fact that $f^{\vee} \geq 0$ on $\left.E \times E^{*}\right)$ that

$$
f^{\doteqdot}(x) \vee 0 \leq \inf _{t^{*} \in E^{*}} f^{\vee}\left(x, t^{*}\right)
$$

To simplify expressions, let $N:=f^{\div}(x) \vee 0$. Then the definition of $f^{\div}$implies that

$$
\begin{aligned}
\left(y, y^{*}\right) \in E \times E^{*} & \Longrightarrow \quad\left\langle x, y^{*}\right\rangle-f^{@}\left(y, y^{*}\right) \leq N(1+\|y\|) \\
& \Longrightarrow \quad f^{@}\left(y, y^{*}\right)+g\left(y, y^{*}\right) \geq 0,
\end{aligned}
$$


where $g: E \times E^{*} \mapsto \mathbb{R}$ is defined by $g\left(y, y^{*}\right):=N(1+\|y\|)-\left\langle x, y^{*}\right\rangle$. From the Fenchel duality theorem for locally convex spaces (which can be applied since $f^{@}$ is proper and convex and $g$ is $\mathcal{N} \mathcal{W}$-continuous on $E \times E^{*}$; see Zălinescu, 8, Theorem 2.8.3(iii), p. 123]) and (1.1.1), there exists $\left(t, t^{*}\right) \in E \times E^{*}$ such that

$$
f\left(t, t^{*}\right)+g^{@}\left(-t,-t^{*}\right)=f^{@ @}\left(t, t^{*}\right)+g^{@}\left(-t,-t^{*}\right) \leq 0 .
$$

By direct computation,

$$
g^{@}\left(-t,-t^{*}\right)= \begin{cases}-N & \left(t=x \text { and }\left\|t^{*}\right\| \leq N\right) ; \\ \infty & (\text { otherwise }) .\end{cases}
$$

Thus $t=x$ and $\left\|t^{*}\right\| \leq N$, and (1.2.3) gives $f\left(t, t^{*}\right)-N \leq 0$. So we have $f^{\vee}\left(x, t^{*}\right)=$ $f^{\vee}\left(t, t^{*}\right):=f\left(t, t^{*}\right) \vee\left\|t^{*}\right\| \leq N:=f^{\div}(x) \vee 0$. (1.2.1) now follows by combining this with (1.2.2).

Theorem 1.3. Let $E$ be a nonzero real Banach space and let $f: E \times E^{*} \mapsto$ ]$-\infty, \infty]$ be proper, convex and $\mathcal{N W}$-lower semicontinuous. Then $\operatorname{pr}_{1} \operatorname{dom} f=$ $\operatorname{dom} f^{\div}$.

Proof. If $t^{*} \in E^{*}$, then $x \in \operatorname{dom} f^{\div} \Longleftrightarrow f^{\div}(x)<\infty \Longleftrightarrow f^{\div}(x) \vee 0<\infty$. Thus, from Lemma 1.2, $x \in \operatorname{dom} f^{\div} \Longleftrightarrow$ there exists $t^{*} \in E^{*}$ such that $f^{\vee}\left(x, t^{*}\right)<$ $\infty \Longleftrightarrow x \in \operatorname{pr}_{1} \operatorname{dom} f^{\vee} \Longleftrightarrow x \in \operatorname{pr}_{1} \operatorname{dom} f$.

\section{FITZPATRICK FUNCTIONS}

Let $S: E \rightrightarrows E^{*}$ be monotone with graph

$$
G(S):=\left\{\left(x, x^{*}\right) \in E \times E^{*}: x^{*} \in S x\right\} \neq \emptyset .
$$

We define the Fitzpatrick function $\left.\left.\varphi_{S}: E \times E^{*} \mapsto\right]-\infty, \infty\right]$ associated with $S$ by

$$
\varphi_{S}\left(x, x^{*}\right):=\sup _{\left(s, s^{*}\right) \in G(S)}\left[\left\langle s, x^{*}\right\rangle+\left\langle x, s^{*}\right\rangle-\left\langle s, s^{*}\right\rangle\right] .
$$

(The function $\varphi_{S}$ was introduced by Fitzpatrick in [2, Definition 3.1, p. 61] under the notation $L_{S}$.) The monotonicity of $S$ and (2.0.1) imply that

$$
\left(x, x^{*}\right) \in G(S) \Longrightarrow \varphi_{S}\left(x, x^{*}\right)=\left\langle x, x^{*}\right\rangle,
$$

and so $\varphi_{S}$ is proper, convex and $\mathcal{N} \mathcal{W}$-lower semicontinuous.

We use the standard notation $D(S):=\{x \in E: \quad S x \neq \emptyset\}=\operatorname{pr}_{1} G(S)$. (2.0.2) implies that $G(S) \subset \operatorname{dom} \varphi_{S}$, from which $D(S) \subset \operatorname{pr}_{1} \operatorname{dom} \varphi_{S}$.

If $S$ is maximal monotone, then (2.0.2) can be strengthened to the two statements

$$
\left(x, x^{*}\right) \in E \times E^{*} \quad \Longrightarrow \quad \varphi_{S}\left(x, x^{*}\right) \geq\left\langle x, x^{*}\right\rangle
$$

and

$$
\varphi_{S}\left(x, x^{*}\right)=\left\langle x, x^{*}\right\rangle \Longleftrightarrow\left(x, x^{*}\right) \in G(S) .
$$

(See [2, Corollary 3.9, p. 62].)

Let $S: E \rightrightarrows E^{*}$ be monotone. Then we see from $(2.0 .2)$ that, for all $\left(y, y^{*}\right) \in$ $E \times E^{*}$,

$$
\left\{\begin{aligned}
\varphi_{S}\left(y, y^{*}\right) & =\sup _{\left(s, s^{*}\right) \in G(S)}\left[\left\langle s, y^{*}\right\rangle+\left\langle y, s^{*}\right\rangle-\left\langle s, s^{*}\right\rangle\right] \\
& =\sup _{\left(s, s^{*}\right) \in G(S)}\left[\left\langle s, y^{*}\right\rangle+\left\langle y, s^{*}\right\rangle-\varphi_{S}\left(s, s^{*}\right)\right] \\
& \leq \sup _{\left(x, x^{*}\right) \in E \times E^{*}}\left[\left\langle x, y^{*}\right\rangle+\left\langle y, x^{*}\right\rangle-\varphi_{S}\left(x, x^{*}\right)\right] \\
& =\varphi_{S}{ }^{@}\left(y, y^{*}\right) .
\end{aligned}\right.
$$


Let $T$ be a maximal monotone extension of $S$. Clearly, $\varphi_{T} \geq \varphi_{S}$, and so $\varphi_{T}{ }^{@} \leq$ $\varphi_{S}{ }^{@}$. (2.0.5) implies that $\varphi_{T} \leq \varphi_{T}{ }^{\circledR}$, consequently $\varphi_{T} \leq \varphi_{S}{ }^{\circledR}$. It now follows from (2.0.3) and the maximality of $T$ that

$$
\left(x, x^{*}\right) \in E \times E^{*} \quad \Longrightarrow \quad \varphi_{S}{ }^{@}\left(x, x^{*}\right) \geq\left\langle x, x^{*}\right\rangle .
$$

If $\left(s, s^{*}\right) \in G(S)$ and $\left(x, x^{*}\right) \in E \times E^{*}$, then, from (2.0.1), $\left\langle s, x^{*}\right\rangle+\left\langle x, s^{*}\right\rangle-\left\langle s, s^{*}\right\rangle \leq$ $\varphi_{S}\left(x, x^{*}\right)$, and so $\left\langle x, s^{*}\right\rangle+\left\langle s, x^{*}\right\rangle-\varphi_{S}\left(x, x^{*}\right) \leq\left\langle s, s^{*}\right\rangle$. Taking the supremum over $\left(x, x^{*}\right)$, we have proved that $\varphi_{S}{ }^{\circledR}\left(s, s^{*}\right) \leq\left\langle s, s^{*}\right\rangle$. Thus, combining this with (2.0.6),

$$
\left(s, s^{*}\right) \in G(S) \quad \Longrightarrow \quad \varphi_{S}{ }^{\circledR}\left(s, s^{*}\right)=\left\langle s, s^{*}\right\rangle .
$$

Lemma 2.1. Let $E$ be a nonzero real Banach space, let $S: E \rightrightarrows E^{*}$ be maximal monotone and $z \in \operatorname{int}\left(\operatorname{pr}_{1} \operatorname{dom} \varphi_{S}\right)$. Then there exist $K>0$ and $\left.\left.\eta \in\right] 0,1\right]$ such that

$$
\left\{\begin{aligned}
\|s-z\| \leq \eta & \text { and }\left(y, y^{*}\right) \in E \times E^{*} \\
& \Longrightarrow \varphi_{S}{ }^{\circledR}\left(y, y^{*}\right)+K\|y-s\|-\left\langle s, y^{*}\right\rangle \geq \eta\left(\left\|y^{*}\right\|-K\right) .
\end{aligned}\right.
$$

Further,

$$
\|s-z\| \leq \eta \quad \Longrightarrow \quad s \in D(S) .
$$

Proof. Theorem 1.3 implies that $z \in \operatorname{int}\left(\operatorname{dom} \varphi_{S} \div\right)$. Since $\varphi_{S} \div$ is proper, convex and lower semicontinuous, it follows from Rockafellar, 4, Corollary 7C, p. 61] (see also Moreau, [3, Proposition 5.f, p. 30] or [6, Lemma 12.2, p. 28] for simpler proofs of Rockafellar's result) that $\varphi_{S} \div$ is continuous at $z$. Let $N=\varphi_{S} \div(z) \vee 0+1$. Then there exists $\eta \in] 0,1]$ such that

$$
x \in E \text { and }\|x\| \leq 2 \eta \quad \Longrightarrow \quad \varphi_{S} \div(x+z) \leq N .
$$

Let $\left(y, y^{*}\right)$ be an arbitrary element of $E \times E^{*}$. Then we have

$$
x \in E \text { and }\|x\| \leq 2 \eta \quad \Longrightarrow \quad\left\langle x+z, y^{*}\right\rangle-\varphi_{S}{ }^{@}\left(y, y^{*}\right) \leq N(1+\|y\|) .
$$

Taking the supremum over $x$, we obtain

$$
\varphi_{S}{ }^{\circledR}\left(y, y^{*}\right)-\left\langle z, y^{*}\right\rangle+N(1+\|y\|) \geq 2 \eta\left\|y^{*}\right\|,
$$

from which

$$
\|s-z\| \leq \eta \quad \Longrightarrow \quad \varphi_{S}{ }^{\circledR}\left(y, y^{*}\right)-\left\langle s, y^{*}\right\rangle+N(1+\|s\|+\|y-s\|) \geq \eta\left\|y^{*}\right\| .
$$

Thus, since $\eta \leq 1$,

$$
\begin{aligned}
\|s-z\| \leq \eta & \Longrightarrow \varphi_{S}{ }^{\circledR}\left(y, y^{*}\right)-\left\langle s, y^{*}\right\rangle+N(2+\|z\|+\|y-s\|) \geq \eta\left\|y^{*}\right\| \\
& \Longrightarrow \varphi_{S}{ }^{\circledR}\left(y, y^{*}\right)-\left\langle s, y^{*}\right\rangle+N\|y-s\| \geq \eta\left\|y^{*}\right\|-(2+\|z\|) N .
\end{aligned}
$$

(2.1.1) now follows by setting $K:=(2+\|z\|) N / \eta \geq N$. Let $s \in E$ and $\|s-z\| \leq \eta$. Then, from (2.1.1),

(2.1.3) $\left(y, y^{*}\right) \in E \times E^{*} \quad \Longrightarrow \quad \varphi_{S}{ }^{\circledR}\left(y, y^{*}\right)+K\|y-s\|-\left\langle s, y^{*}\right\rangle \geq \eta\left(\left\|y^{*}\right\|-K\right)$.

We next show that

$$
\left(y, y^{*}\right) \in E \times E^{*} \quad \Longrightarrow \quad \varphi_{S}{ }^{\circledR}\left(y, y^{*}\right)+K\|y-s\|-\left\langle s, y^{*}\right\rangle \geq 0 .
$$

To this end, let $\left(y, y^{*}\right) \in E \times E^{*}$. (2.1.4) is immediate from (2.1.3) if $\left\|y^{*}\right\| \geq K$. If, on the other hand, $\left\|y^{*}\right\|<K$, then, from (2.0.6),

$\varphi_{S}{ }^{@}\left(y, y^{*}\right)+K\|y-s\|-\left\langle s, y^{*}\right\rangle \geq K\|y-s\|+\left\langle y-s, y^{*}\right\rangle \geq K\|y-s\|-\|y-s\|\left\|y^{*}\right\| \geq 0$, 
which completes the proof of (2.1.4). (2.1.4) implies that $\varphi_{S}{ }^{@}+g \geq 0$ on $E \times E^{*}$, where $g: E \times E^{*} \mapsto \mathbb{R}$ is defined by $g\left(y, y^{*}\right):=K\|y-s\|-\left\langle s, y^{*}\right\rangle$. From the Fenchel duality theorem for locally convex spaces (which can be applied, as before, since $f^{@}$ is proper and convex and $g$ is $\mathcal{N} \mathcal{W}$-continuous on $\left.E \times E^{*}\right)$ and (1.1.1), there exists $\left(t, t^{*}\right) \in E \times E^{*}$ such that

$$
\varphi_{S}\left(t, t^{*}\right)+g^{@}\left(-t,-t^{*}\right)=\varphi_{S}{ }^{@}\left(t, t^{*}\right)+g^{@}\left(-t,-t^{*}\right) \leq 0 .
$$

By direct computation,

$$
g^{@}\left(-t,-t^{*}\right)= \begin{cases}-\left\langle s, t^{*}\right\rangle & \left(t=s \text { and }\left\|t^{*}\right\| \leq K\right) ; \\ \infty & \text { (otherwise). }\end{cases}
$$

Thus $t=s$ and $\left\|t^{*}\right\| \leq K$, and (2.1.4) gives us that $\varphi_{S}\left(s, t^{*}\right)-\left\langle s, t^{*}\right\rangle \leq 0$. (2.0.3) and (2.0.4) now imply that $\left(s, t^{*}\right) \in G(S)$, from which $s \in D(S)$. This gives (2.1.2), and completes the proof of Lemma 2.1 .

Theorem 2.2. Let $E$ be a nonzero real Banach space and let $S: E \rightrightarrows E^{*}$ be maximal monotone. Then

$$
\operatorname{int}\left(\operatorname{pr}_{1} \operatorname{dom} \varphi_{S}\right)=\operatorname{int} D(S) .
$$

In particular, int $D(S)$ is convex.

Proof. It is clear from Lemma 2.1 that $\operatorname{int}\left(\operatorname{pr}_{1} \operatorname{dom} \varphi_{S}\right) \subset \operatorname{int} D(S)$. The reverse inclusion is obvious since $D(S) \subset \operatorname{pr}_{1} \operatorname{dom} \varphi_{S}$.

Remark 2.3. In the proof of Lemma 2.1, we did not use the full force of the cited result of Rockafellar, which is, in fact, valid if $z$ is an internal point (rather than an interior point) of $\operatorname{dom} \varphi_{S} \div$. Using this fact, one can easily generalize Theorem 2.2 and obtain results similar to the "six set theorem" and "nine set theorem" of [6. Theorems 18.3-4, p. 50] without having to use an abstract "big convexification".

\section{REFERENCES}

[1] J. M. Borwein, Maximal monotonicity via convex analysis, Preprint.

[2] S. P. Fitzpatrick, Representing monotone operators by convex functions, Workshop/ Miniconference on Functional Analysis and Optimization, Proc. Centre Math. Anal. Austral. Nat. Univ., vol. 20, Austral. Nat. Univ., Canberra, 1988, pp. 59-65. MR1009594 (90i:47054)

[3] J.-J. Moreau, Fonctionelles convexes, Collège de France, Séminaire sur les équations aux derivées partielles, Paris, 1966.

[4] R. T. Rockafellar, Level sets and continuity of conjugate convex functions, Trans. Amer. Math. Soc. 123 (1966), 46-63. MR0192318 (33:544)

[5] R. T. Rockafellar, Local boundedness of Nonlinear, Monotone Operators, Michigan Math. J. 16 (1969), 397-407. MR0253014 (40:6229)

[6] S. Simons, Minimax and monotonicity, Lecture Notes in Mathematics, vol. 1693, SpringerVerlag, 1998. MR $1723737(2001 \mathrm{~h}: 49002)$

[7] S. Simons and C. Zălinescu, Fenchel duality, Fitzpatrick functions and maximal monotonicity, J. of Nonlinear and Convex Anal. 6 (2005), 1-22. MR.2138099

[8] C. Zălinescu, Convex analysis in general vector spaces, World Scientific, 2002. MR1921556 (2003k:49003)

Department of Mathematics, University of California, Santa Barbara, California 93106-3080

E-mail address: simons@math.ucsb.edu 